\title{
Billroth II Procedure
}

National Cancer Institute

\section{Source}

National Cancer Institute. Billroth II Procedure. NCI Thesaurus. Code C51582.

Surgical removal of the lower part of the stomach with end-to-end anastomosis of the remaining segment of the stomach with the jejunum; in this procedure the duodenum is closed off. 